\title{
CONSIDERACIONES GENERALES EN TORNO A LA COMPRENSIÓN DE LA REALIDAD DEL MUNDO SOCIAL
}

General Considerations around the Understanding of the Reality of the Social World

\author{
Saúl Marcelo Chinche Calizaya
}

Universidad Mayor de San Simón, Bolivia

\begin{tabular}{ll} 
KEY WORDS & ABSTRACT \\
\cline { 2 - 3 } $\begin{array}{l}\text { Doxa } \\
\text { Praxis }\end{array}$ & $\begin{array}{l}\text { The construction of knowledge in the Social Sciences, undoubtedly has an } \\
\text { unfinished character given its provisional nature; It is clear that there is }\end{array}$ \\
Intersubjectivity & always something radically incomplete in this type of knowledge, whose \\
Lebenswelt & study is precisely oriented to the analysis of social phenomena. We arrive \\
Classifications & at this statement, by virtue of the fact that social phenomena in general \\
Reflectivity & tend to resemble unrepeatable periodicities not only because of their \\
& $\begin{array}{l}\text { actors who interact in it, but basically because of the delimitation of } \\
\text { time-space; added to the fact that they are unstable, complex, dynamic } \\
\text { and contradictory, which make it even more difficult to understand and } \\
\text { interpret them. }\end{array}$
\end{tabular}

PALABRAS CLAVE

Doxa

Praxis

Fenomenología

Intersubjetividad

Lebenswelt

Tipificaciones

Reflexividad

\section{RESUMEN}

La construcción de conocimiento en las Ciencias Sociales, posee sin duda alguna, un carácter inacabado dada su provisoriedad; pues queda claro que existe siempre algo radicalmente incompleto en este tipo de conocimiento, cuyo estudio se halla precisamente orientado al análisis de los fenómenos sociales. Arribamos a esta afirmación, en virtud de que los fenómenos sociales en general, tienden a asemejarse a periodicidades irrepetibles no sólo por sus actores que en ella interactúan, sino básicamente por la delimitación del tiempo-espacio; sumados al hecho de que son inestables, complejas, dinámicas y contradictorias, que dificultan aún más, las posibilidades de su aprehensión e interpretación.

Recibido: $13 / 08 / 2020$

Aceptado: 20/11/2020 


\section{Introducción}

$\mathrm{E}$ l presente trabajo pretende apenas abordar teóricamente algunos aspectos que permitan clarificar la comprensión de la realidad del mundo social. Para tal efecto partimos del supuesto de que el conocimiento en las Ciencias Sociales posee un carácter inacabado dado su provisoriedad, ratificando con ello que existe siempre algo radicalmente incompleto en este tipo de conocimiento.

La justificación a esta postura inicial, estriba en la lógica de que los fenómenos sociales en general, tienden a asemejarse a periodicidades irrepetibles no sólo por sus actores que en ella interactúan, sino básicamente por la delimitación del tiempo-espacio, sumados a que éstos son inestables, complejas, dinámicas, contradictorias y que dificultan aún más las posibilidades de su aprehensión e interpretación.

Si bien, por un lado, esto trae consigo profundas preocupaciones a la hora de realizar abordajes sobre la realidad -concretamente sobre los fenómenos sociales-; por otro, tienden a generar interesantes desafíos y puntos de partida orientadores en su análisis, que permiten vislumbrar que éstos difícilmente pueden ser similares, aún si contaran con características morfológicas comunes (comprensión de la totalidad del objeto), el análisis de aquellos aspectos que a simple vista no pueden ser apreciados (circunstancias, intencionalidades, orientaciones propias de los actores) y, quizá la más importante, los fenómenos sociales nunca se repiten tal como ya han ocurrido, pues los actores, las circunstancias, intenciones y orientaciones que guiaron la acción ya no son los mismos (carácter histórico).

Por ende, las conclusiones que formulemos con relación a los fenómenos sociales observados, no pueden ser sino provisionales; pues alcanzar la comprensión en su totalidad de los mismos, es poco menos que imposible dada su característica y naturaleza (realidad de la vida) que nos circunda y en la cual, el cientista social se halla inmerso en su especificidad, obligándole a desplegar esfuerzos cognitivos de comprensión que vayan más allá de los aspectos visibles de la acción, hasta tocar aquello que la inspira. Vale decir, su sentido subjetivo y mentado; tarea que pasa necesariamente por retomar en el marco de una opción teórica determinada, múltiples elementos que consideren directamente aquellos planos de la empiria de la vida social.

Dicho de otra forma, abordar tales implicancias resultan ser por un lado, altamente gratificantes para los cientistas sociales, pues se hallan ante la imperiosa necesidad de dar cuenta de una realidad compartida que es particular y específica, pero al mismo tiempo cambiante y dinámica.

Por otro lado, abre la posibilidad de que esta realidad pueda ser comprendida, interpretada y captada desde la propia experiencia, aunque no del todo individual; esto en razón a que la experiencia misma del mundo, es una genuina experiencia existencial colectiva de ese mundo cotidiano; que se construye y reconstruye, se configura y reconfigura en la dinámica de esas interacciones situadas y fechadas, en las significaciones y resignificaciones colectivas. Es ahí precisamente, donde radica su valía y trascendencia.

\section{DESARROLLO}

\subsection{Aspectos Generales}

La construcción de conocimiento en las Ciencias Sociales, posee sin duda alguna, un carácter inacabado dada su provisoriedad ${ }^{1}$; pues queda claro que existe siempre algo radicalmente incompleto en este tipo de conocimiento, cuyo estudio se halla precisamente orientado al análisis de los fenómenos sociales².

\footnotetext{
${ }^{1}$ Al respecto conviene aclarar que esta provisoriedad bajo ninguna circunstancia debe entendérsela como una precariedad científica, sino más bien como el reconocimiento tácito de aquello inacabado, de la necesidad de continuidad del debate, de repensar y reflexionar lo afirmado y, sobre todo, de la posibilidad -inclusive- de retroceder o seguir avanzando en la construcción del conocimiento.

2 Sin el ánimo de realizar constructos conceptuales profundos con relación a los fenómenos sociales, conviene señalar que comprender los mismos exige ir más allá de lo objetivado (lo real, lo concreto observado a primera vista); pues en muchos casos, se requiere apelar a la intersubjetividad -que implica la posibilidad de reconocer que en esa realidad estudiada nos encontramos inmersos, vivimos en ella, pero también nosotros construimos esa realidad compartida, la cual nos es común a todos porque compartimos el mismo tiempo y espacio con otros-, para entender las razones o circunstancias que explican las acciones que en ella se observan. Dicho de otra forma, se requiere ir más allá de los aspectos visibles de la acción, y hasta quizás, como decía Peter Berger, mirar "tras las fachadas" de las estructuras sociales que se nos presentan en la realidad.
} 
Arribamos a esta afirmación, en virtud de que los fenómenos sociales en general, tienden a asemejarse a periodicidades irrepetibles no sólo por sus actores que en ella interactúan, sino básicamente por la delimitación del tiempoespacio; sumados al hecho de que son inestables, complejas, dinámicas y contradictorias, que dificultan aún más, las posibilidades de su aprehensión e interpretación.

Si bien, por un lado, esto trae consigo profundas preocupaciones a la hora de realizar abordajes sobre la realidad $\mathrm{y}$, concretamente sobre los fenómenos sociales; por otro, tiende a generar interesantes desafíos y puntos de partida orientadores en su análisis, ya que permiten comprender que éstos difícilmente pueden ser similares, aún si contaran con características morfológicas comunes (comprensión de la totalidad del objeto), el análisis de aquellos aspectos que a simple vista no pueden ser apreciados (circunstancias, intencionalidades, orientaciones propias de los actores) y, quizá la más importante, los fenómenos sociales nunca se repiten tal como ya han ocurrido, pues los actores, las circunstancias, intenciones y orientaciones que guiaron la acción ya no son los mismos (carácter histórico).

A esto, bien podríamos denominar como la historicidad de los hechos y que la Sociología reconoce como un importante artefacto (instrumento) para el análisis y comprensión de la realidad; los fenómenos sociales y su interacción basado en normas y leyes del desarrollo social, valorando su especificidad natural y originalidad; pero al mismo tiempo de continua modificación y cambio, hacen que los fenómenos jamás se repitan de la misma forma como ya ocurrieron anteriormente (delimitación temporo-espacial sociohistórica).

Por ende, las conclusiones que formulemos con relación a los fenómenos sociales observados, no pueden ser sino provisionales; pues alcanzar la comprensión en su totalidad de los mismos, es poco menos que imposible dada su característica y naturaleza (realidad de la vida) que nos circunda y en la cual, el cientista social se halla inmerso en su especificidad, obligándole a desplegar esfuerzos cognitivos de comprensión que vayan más allá de los aspectos visibles de la acción -siguiendo con ello el discurso Weberiano- hasta tocar aquello que la inspira. Vale decir, su sentido subjetivo, mentado -que Alfred Schutz tanto buscó traspasar-; tarea que pasa necesariamente por retomar en el marco de una opción teórica determinada, múltiples elementos que consideren directamente aquellos planos de la empiria de la vida social.

Dicho de otra forma, abordar tales implicancias resultan ser por un lado, altamente gratificantes para los cientistas sociales, pues se hallan ante la imperiosa necesidad de dar cuenta de una realidad compartida que es particular y específica, pero al mismo tiempo cambiante y dinámica.

Por otro lado, abre la posibilidad de que esta realidad pueda ser comprendida, interpretada y captada desde la propia experiencia, aunque no del todo individual; esto en razón a que la experiencia misma del mundo, es una genuina experiencia existencial colectiva de ese mundo cotidiano $^{3}$; que se construye y reconstruye, se configura y reconfigura en la dinámica de esas interacciones situadas $y$ fechadas, en las significaciones y resignificaciones colectivas. Es ahí precisamente, donde radica su valía y trascendencia.

Bajo esa orientación, sólo se puede comprender la "lógica más profunda del mundo social a condición de sumergirse en la particularidad de una realidad empírica, históricamente situada y fechada, pero para elaborarla como caso particular de lo posible,..... es decir, como caso de figura en un universo finito de configuraciones posibles" (Bourdieu, 1997, 12).

Esto sin duda, amplia aún más la preocupación latente del peligro y exposición a los embates de oposiciones y refutaciones de los seguidores de la doxa tradicional positivista y dogmática que sistemáticamente desterraron de sus postulados, el hecho de asumir que el conocimiento de la realidad es simple y

\footnotetext{
3 De hecho, la Sociología fenomenológica se ocupa de analizar el mundo cotidiano, otorgando especial interés y preocupación a aquellas formas, maneras y dispositivos que utilizan las personas en la vida cotidiana para la construcción y producción de significados. Cabe señalar, que si bien, el surgimiento de la fenomenología le reconoce a Edmund Husserl como uno de sus máximos exponentes, ocupa también un sitial privilegiado -y hasta en algunos casos preponderante, esto en el campo de las Ciencias Sociales-, la figura de Alfred Schutz, quién intenta otorgarle a la fenomenología un carácter más próximo al análisis y estudio sociológico de la realidad social.
} 
llanamente, un conjunto de conocimientos provisionales acerca de los fenómenos sociales.

Peor aún, si planteamos el hecho de asumir como punto de partida, la búsqueda y construcción de un pensamiento (doxa) no definitivo, incompleto, menos totalitario; y que en ese afán de hallar mayores niveles de credibilidad y solidez a nuestros pensamientos, permanentemente -los cientistas sociales-, nos encontramos teóricamente afrontados y confrontados (posicionamientos epistemológicos sustentados desde enfoques diversos), ante una suerte de relatividad e inestabilidad teórica, susceptible de transformación y/o cambio aunque no por ello, carente de rigurosidad teórica y metodológica-, en la comprensión de los fenómenos sociales, las cuales se van desarrollando en sociedades caracterizadas por su particular historicidad.

De ahí, que en el caso de las Ciencias Sociales, resulte esencial:

ofrecer unos instrumentos de conocimiento que puedan volverse contra el sujeto del conocimiento, no para destruir o desacreditar el conocimiento (científico), sino, por el contrario, para controlarlo y reforzarlo. (Bourdieu, 2003, 17)

En otras palabras, una aparente objetivación en las Ciencias Sociales, no es otra cosa que una acción de objetivación bastante sui generis, que se halla construida sobre la base de una rigurosidad intelectual -lo cual no puede negarse bajo ninguna circunstancia-, guiados por el uso de métodos y técnicas que facilitan el hallazgo de nuevos conocimientos en el campo científico-social.

\subsection{Realidad Social: Algunas Reflexiones Analíticas}

Por lo general, la concepción misma de realidad del mundo tiene un carácter de comprensión insuficiente y hasta en algunos casos sesgada, en virtud de que se le atribuye sólo a los sentidos, la posibilidad de abordar la realidad (objetos), pero que deja de lado, a aquellas otras formas, estados o situaciones existentes en la realidad y que no son accesibles a los sentidos orgánicos; al extremo tal, que ni aún las ciencias ni la reflexión filosófica -concretamente la gnoseología-, han logrado enfocar con cierta precisión e integralidad, la naturaleza y complejidad de sus componentes.

El positivismo en cuanto ideología, planteaba que el desarrollo de las ciencias de principios del siglo XX, constituían el logro máximo y definitivo del conocimiento objetivo y fiable.

$\mathrm{Si}$ bien, el término positivismo posee múltiples acepciones, generalmente se la utiliza para "referirse a la búsqueda de las leyes invariantes del mundo natural, así como del social", las cuales se obtienen a partir de la "investigación sobre el mundo social y/o de la teorización sobre ese mundo" (Ritzer, 1993, 93).

Conviene sin embargo, tener presente que el positivismo se inaugura en base al error histórico de separar sujeto-objeto. Es decir, negando radicalmente la existencia de la relación entre el sujeto y el objeto; además de considerar que los hechos derivan única y exclusivamente de la observación y la experimentación, y que pueden ser analizados de forma neutra y objetiva (investigación empírica) ${ }^{4}$.

De esta forma, los positivistas "se plantean las cuestiones con el único objetivo de averiguar el verdadero estado de las cosas, y reproducirlo con la mayor precisión en sus teorías.....", las cuales adquieren validez en la "medida en que constituye una representación exacta y completa de las relaciones que existen naturalmente" (Ritzer, 1993, 93); algo así como una copia o una teoría de la copia de la verdad.

Bajo ese planteamiento, el espíritu de la ciencia debe servirse de la observación directa, la comprobación y la experiencia -y en el fondo, de la investigación empírica-, como procedimientos que otorguen valor y rigurosidad científica.

Algunos argumentos fuertes planteados por el positivismo, señalan que el origen de las ciencias -y el conocimiento como tal-, es propio de cada individuo, en razón a que es precisamente esa $\boldsymbol{s u}$ experiencia la que otorga significatividad al conocimiento.

\footnotetext{
${ }^{4}$ Parafraseando a Comte, quién considera que la única posibilidad de explicación del mundo natural y/o social y su teorización, es posible sólo a través de la investigación como medio para descubrir las leyes de aplicación a ese mundo social y/o natural; pero que en ese proceso no se excluye a la investigación empírica la cual debe hallarse subordinada a la teoría. De esta forma, construye una máxima positivista que refiere que sólo existen dos caminos para alcanzar el mundo real que existe fuera de la mente: Investigación y teorización.
} 
Posteriormente, cuando esa experiencia es compartida, reflexionada y estudiada por una colectividad -en este caso, una comunidad científica-, viene a convertirse en $\mathbf{l a}$ experiencia que es aceptada por esa comunidad científica que, progresivamente transformará esos datos observados en datos numéricos, para luego dar paso, a la construcción de instrumentos, cuya finalidad última sea la socialización, difusión y reproducción de información especializada sobre ese conocimiento $\mathrm{y}$, principalmente, formular leyes y teorías de alcance general.

A decir de los positivistas, la "teoría es la única capaz de comprender y explicar las condiciones en las que se dan los procesos de construcción de conocimientos en los otros modos de apropiación" (Massé Narvaez, 2001, 9).

Sin embargo, esa forma de concebir el conocimiento -de acuerdo a las características descritas líneas arriba-, sólo puede hallarse en el análisis de los hechos reales, los mismos que deben ser objeto de rigurosas descripciones lo más neutrales, objetivas y completas posibles.

Estos elementos constituyen prerrequisitos aplicables en todas las etapas de construcción del conocimiento, por cuanto los hechos reales fácilmente pueden ser calificados de verdaderos o falsos, haciendo que su cuantificación -las más de las veces matematizado-, sea relativamente sencilla (objetiva).

Con relación al observador-investigador, se le exige liberarse de todo posicionamiento personal de subjetividad -ideas precipitadas, sentimientos y apasionamientos-, respecto al hecho observado (neutralidad). En lo referente a la reproducción, difusión de información y consecuente formulación de leyes de alcance general, éstas adquieren validez en tanto se hallan sustentadas en la observación sistemática de los hechos sensibles (descripción completa).

De lo que se trata, es de captar una "realidad objetiva totalmente inaccesible a la experiencia común por el análisis de las relaciones estadísticas entre distribuciones de propiedades materiales, expresiones cuantificadas de la repartición del capital (en sus diferentes especies) entre los individuos en competencia por su apropiación" (Bourdieu, 2007, 218).

El positivismo plantea que el desarrollo de la ciencia se sustenta en el logro máximo y definitivo del conocimiento objetivo y fiable; requiriendo para ello, un conjunto de principios teóricos y características técnico-metodológicas específicas, referidas a áreas de realidad muy determinadas -delimitación en tiempo y espacio, y que se encuentran precisamente reservadas a la capacidad de accesibilidad por los sentidos orgánicos.

Inferir que la realidad en su totalidad es comprendida y descrita a partir de los sentidos, constituye -a nuestro modo de ver-, sólo una posibilidad de exploración forzada, incluso arbitraria y profunda de la dinámica que subyace en todo aquello que los sentidos perciben como objetos, sobre los cuales se construyen una especie de modelos lógico-explicativos de esa realidad, plasmados en constructos conceptuales que se basan en representaciones.

Tales representaciones, son irreales en razón a que se remiten exclusivamente a todo aquello que es captado por los sentidos orgánicos que poseen los seres humanos; pero que dejan de lado, aquellos otros estados o situaciones también existentes en la realidad, que no son fácilmente accesibles a los sentidos orgánicos de la especie humana.

Precisamente, sobre estos estados y situaciones que orientan las acciones e intenciones de los seres humanos y que son inaccesibles a los sentidos, se han ido elaborando un conjunto de metodologías y técnicas para registrar y descifrar todas aquellas "significaciones que los agentes producen como tales por una percepción diferencial de esas mismas propiedades, constituidas así como signos distintivos... mediante los cuales los agentes clasifican y se clasifican"(Bourdieu, 2007, 218), asumiéndose inmersos en la especificidad y naturaleza de "ese" o "esos" fenómenos particulares, que revisten especial interés y preocupación para una determinada comunidad científica.

Al parecer, las ciencias aciertan en algún modo, guiados por una serie de procedimientos técnicos para brindar un panorama parcial de la realidad, pero que definitivamente -dadas sus limitaciones de origen-, resulta imposible proporcionar una visión completa de la totalidad de la realidad. 
Una reflexión inicial construida a partir de lo señalado líneas arriba, se halla referido al hecho de que cualquier análisis acerca del mundo-real, debe considerar que éste se encuentra indeterminado.

Esto, en razón a que no existe posibilidad lineal de que el ser humano se encuentre automáticamente preprogramado por naturaleza, para conocer, pensar, valorar, optar y organizar la conducta y la convivencia del modo más lógico posible, para dar cuenta de la realidad que percibe, recurriendo para ello, a la clásica separación mente-cosas; además de la discusión clásica acerca de la existencia o no existencia de relación entre el sujeto y el objeto de estudio.

$\mathrm{Al}$ respecto, consideramos que una posibilidad de superación a esta clásica indeterminación, pasa por dejar de lado, la "rígida dicotomía mente/cosas y admitir una serie de tipos y modos de darse la realidad, intermedios entre la vida psíquica... y la cristalización última de las supuestas cosas, tal como la mente las percibe en su cotidianeidad" (Ortíz-Osés; Lanceros, 2006, 471).

De hecho, la primera aproximación de lo inmediato a la experiencia humana, son las vivencias como tal -no así las cosas o las sensaciones orgánicas que éstas provocan-, y más concretamente la praxis donde los sujetos se encuentran afectados por una serie de intereses y estímulos, producto de la interacción con el objeto -que no precisa exclusivamente que éstos se circunscriban a situaciones y/o acciones captadas por los sentidos-, que a su vez, son condicionados por una serie de relaciones y comportamientos.

De ahí que la "relación de conocimiento entre sujeto y objeto implica la conciencia racional del sujeto que quiere construir conocimiento, pues supone haber pasado por un proceso en el cual el despliegue del objeto permite al sujeto identificar con nitidez el campo de opciones posibles a desarrollar" (Massé Narvaez, 2001, 9).

En ese proceso de aprehensión de la realidad por parte del sujeto no existe nada caótico. Al contrario, todo presenta un orden de prelación de fases, de antecedentes y de consecuencias que se hallan debidamente contextualizados y vinculados a una serie de estados y antecedentes que poseen valor y significado.
Inclusive, estos estados y antecedentes se hallan perpetuados con una identidad determinada en cuanto están integrados en una estructura sistémica y lógica, a la que describimos como objetivos y que hace que sean sujetos de estudio y análisis, al hallarse incluidos en esas estructuras incorporadas a la praxis y la subjetividad humanas.

Bajo esa lógica, el "objeto propiamente dicho del conocimiento humano no es la entidad material de las cosas, sino su significado y su posición dentro de un sistema de coordenadas lógicas y simbólicas a la vez" (Ortíz-Osés; Lanceros, 2006, 472).

Así, lo más cercano a la verdad es que además de esta contextualización, cuando a un objeto se le atribuye realidad o existencia, el referente único es la propia densidad asertiva de quién lo afirma y que atribuye más o menos con algún fundamento, al objeto real que dice conocer.

La realidad "no es el conjunto de todas las cosas ni la interacción de todos los fenómenos naturales o la actividad de las partículas subatómicas, sino la praxis, sus episodios y los productos materiales y culturales que resultan de ella" (Ortíz-Osés; Lanceros, 2006, 477); las cuales necesariamente tienen un cierto significado que los habilita para ser cognoscibles y que sólo llegan a ser conocidas en cuanto se hallan mediatizados por la praxis.

De esta forma, la percepción real del o los objetos, no se agota en las sensaciones ni en el procesamiento cerebral, sino que requiere además de la construcción, el uso y manejo de ciertas estructuras identificables con una determinada función dentro de un sistema de necesidades integradas en algún sistema de objetos, que adquieren relieve cognitivo $y$ trascendencia en la praxis realizada por parte del grupo social -en este caso, el colectivo social-, para constituir los referentes reales de esa praxis.

Tal apreciación, resulta ser coherente con el precepto básico que plantea que las "ciencias no pueden agotar las posibilidades de conocimiento válido, ni de realidad, pues constituyen sólo una de tantas formas de contextualizar la hermenéutica ${ }^{5}$

\footnotetext{
5 La hermenéutica se constituye como una filosofía de la comprensión del ser a través de la interpretación del lenguaje, el cual funge así de mediación y que actúa como interlenguaje o idioma universal de nuestra cultura.
} 
cualificada del mundo... dentro de un espacio geocultural, de un tiempo y de una sociedad" (Ortíz-Osés; Lanceros, 2006, 477)

Con ello, aceptamos abiertamente que la idea de realidad no es, ni debe ser exterior al sujeto que la examina, la analiza y cuestiona; resultando innegable admitir también la existencia de la relación indisoluble entre el sujeto y el objeto, cuyo planteamiento es ampliamente defendido por la fenomenología.

Sin embargo, referirnos a la fenomenología, exige como condición previa, remontarnos a los postulados teóricos del alemán Edmund Husserl (1858-1938), principal exponente de esta corriente filosófica, quién mostró especial preocupación por reposicionar la creatividad, la intención y la esperanza individuales que posibilitan la construcción del orden colectivo.

A decir de Husserl, la "fenomenología significaba que la realidad está estructurada por la percepción. Aún las cosas cuya objetividad damos por sentada están allí sólo porque queremos o hacemos que estén allí" (Alexander, 1992, 196).

Bajo ese planteamiento, la fenomenología científica Husserliana supone un "compromiso de penetrar en los diversos estratos construidos por los actores del mundo social con el fín de alcanzar la estructura esencial de la conciencia, el ego trascedental" (Ritzer, 1993, 368); que no es otra cosa, que ese universo propio de la vida consciente, "la corriente íntegra de pensamiento, con todas sus actividades y sus cogitaciones $y$ experiencias" (Ritzer, 1993, 368).

Con ello, Husserl muestra especial preocupación por las carácterísticas básicas e invariantes de la conciencia humana -pues interesa conocer la esencia, especialmente la esencia de la conciencia del ego trascendental-; la descripción de las estructuras de la experiencia tal y como se presentan en la conciencia, sin recurrir a teorías, deducciones o suposiciones procedentes de otras disciplinas.

De hecho, el núcleo central de la teoría fenomenológica de Husserl, concibe que la conciencia es un proceso que no se encuentra en la mente del sujeto, sino en las relaciones establecidas entre el actor (sujeto) y los objetos existentes en el mundo exterior y es precisamente ahí, en esa relación (sujeto - objeto), donde adquiere intencionalidad. Por lo mismo, la conciencia es siempre conciencia de algo, es decir, de algún objeto.

Este planteamiento rompe de alguna manera, con la tradicional separación del sujeto y el objeto de conocimiento por teorías que hacían valer la preponderancia de uno sobre otro, y que dieron paso al surgimiento de corrientes antagónicas como el subjetivismo y el objetivismo por un lado, y por otro, la construcción de metodologías de pensamiento en las que de alguna manera, es posible lograr determinados grados de integración sujetoobjeto, cual es el caso de la fenomenología.

Precisamente, la fenomenología se coloca por encima de ese antagonismo (objetivismo subjetivismo), a través de la idea de intencionalidad de los procesos de conciencia que posee "una inmensa plenitud de efectuaciones, pero sólo en conexión y en entretejimiento indisoluble con una situación fáctica y, en la referencia cada vez más amplia de las situaciones con un mundo fáctico" (Szilasi, 1973, 52).

Ahora bien, esta intencionalidad no debe ser considerada como un atributo, sino más bien como un componente generador de la conciencia y como tal, siempre se halla dirigida hacia algo, pues toda vivencia, actitud anímica y emotiva se dirige hacia algo concreto. De ahí que la intención puede ser considerada un carácter de ser de la conciencia.

Esta orientación inicial sería retomada aunque sólo en parte-, por el austríaco Alfred Schutz (1899-1959), con algunas precisiones que le otorgan mayor proximidad al estudio de la realidad del mundo social 6 , a la que denominó sociología fenomenológica ${ }^{7}$, del cual es su principal teórico.

\footnotetext{
${ }^{6}$ De hecho, la teoría fenomenológica de Edmund Husserl, centró su preocupación estructural hacia la explicación del ego trascendental considerado como la corriente integra de pensamiento de ese universo propio de la vida consciente, con todas sus actividades y experiencias. En cambio, Alfred Schutz, orientó su trabajo hacia la intersubjetividad exterior, el mundo social y el mundo de la vida cotidiana.

${ }^{7}$ La sociología fenomenológica se desarrolla a partir de premisas un tanto alejadas de los planteamientos filosóficas de Edmund Husserl, aunque en alguna medida las retoma. El debate general se concentra en torno a cómo se puede lograr el conocimiento, y su aparición se sustenta en la comprensión de la fenomenología como instancia de aproximación metodológica a lo cotidiano; marcando distancias notables con aquellas formas de pensamiento de la
} 
Desde un punto de vista epistemológico, podemos mencionar que la fenomenología implica una ruptura con las formas de pensamiento de la sociología tradicional, ya que enfatiza la necesidad de comprender la realidad más que de explicarla; sugiriendo que es en el durante, en el aquí y en el ahora, donde es posible identificar elementos de significación que describen y construyen lo real.

Esto queda plenamente ratificado, en tanto se acepta que la "realidad de la vida cotidiana se organiza alrededor del "aquí" de mi cuerpo y el "ahora" de mi presente. Este "aquí y ahora" es el foco de la atención que presto a la realidad de la vida cotidiana. Lo que "aquí y ahora" se me presenta en la vida cotidiana es lo realissimum de mi conciencia" (Berger; Luckmann, 2001, 39).

Alfred Schutz ${ }^{8}$, muestra especial preocupación y cuidado a la hora de identificar y dar por supuesto sin más, el punto de vista positivista con la ciencia positiva; toda vez que a su modo de ver, existe un tipo de ciencia positiva afín a la fenomenología; el cual se asienta en el terreno empírico para estudiar las situaciones concretas y cotidianas en que se desenvuelve la cultura y la sociedad, pero no es positivista en cuanto no se adhiere al enfoque cognoscitivo del naturalismo y tampoco asume el método característico de las ciencias naturales, restrictivo, estrecho $\mathrm{y}$ cosificado, sino que aspire a ser algo más que una simple técnica que no se doblegue a la tecnocracia.

Con relación al empirismo, conviene mencionar que es según su propio concepto, "una filosofía que ha atribuido a la experiencia la primacía en el orden del conocimiento; pero en verdad.... debido a la falta de autorreflexión del empirismo, en el pensar científico controlado empíricamente, la experiencia no fue tanto

sociología tradicional, ya que enfatiza la necesidad de comprender la realidad, más que de explicarla.

8 Sus principales aportaciones al pensamiento sobre lo social son por un lado, la incorporación del mundo cotidiano a la investigación sociológica a partir de la reivindicación como objeto de estudio de la sociología el ámbito de la sociabilidad. Es decir, el conjunto de las relaciones interpersonales y de las actitudes de la gente que son pragmáticamente reproducidas o modificadas en la vida cotidiana, y por el otro, la definición propia de las características del mundo de la vida, a saber: que sus significados son construcciones sociales; que es intersubjetivo; que está conformado por personas que viven en él con una actitud "natural"; que es un ámbito familiar en el que los sujetos se mueven provistos de un acervo de conocimiento a mano. liberada y desatada cuanto dirigida $y$ encadenada"(Adorno, 2001, 99-100).

Continuando con este análisis, Schutz toma en cuenta decisivamente la cultura y la vida consciente así como los enfoques necesarios para investigarlos, por lo que no se detiene en los elementos fácticos -carácter contingente $\mathrm{y}$ relativo a los hechos-, sino que prolonga la indagación más allá de lo empíricamente evidente, siguiendo la intención fenomenológica de comprender el significado que tienen tales hechos en la vida humana, tanto colectiva como individual y al mismo tiempo, busca elucidar los métodos que emplean los actores sociales para construir las objetividades del mundo social.

Así, la fenomenología es un método que precede a partir del análisis intuitivo de los objetos tal y como son dados a la conciencia, a partir del cual busca inferir los rasgos esenciales de la experiencia y de lo experimentado por los sujetos en el mundo de la vida cotidiana.

Esta conciencia es "siempre intencional, siempre apunta o se dirige a objetos. Nunca podemos aprehender tal o cual substrato supuesto de conciencia en cuanto tal, sino solo la conciencia de esto o aquello. Esto es lo que ocurre, ya sea que el objeto de la conciencia se experimente como parte de un mundo físico exterior, o se aprehenda como elemento de una realidad subjetiva interior" (Berger; Luckmann, 2001, 38).

Al igual que su antecesor -Husserl-, Schutz creía que sólo los fenomenologos "podían obtener un conocimiento absolutamente válido de las estructuras básicas de las experiencias vividas de los actores (especialmente de las conscientes)" (Ritzer, 1993, 368), guiados por una ciencia concebida como filosofía metodológicamente rigurosa y crítica, que permita realizar análisis y descripciones de los fenómenos sociales tal cual son experimentados por los hombres (situaciones, eventos e interacciones sociales).

De ahí que la ciencia de la Sociología, no sólo debe ser capaz de describir el mundo de la vida social, sino también debe ocuparse de ir construyendo modelos teóricos y conceptuales para la comprensión de ese mundo de la vida, que representa la preocupación central de lo que 
él mismo denominó como su sociología fenomenológica9.

Con relación a la ciencia, Schutz la concibe como una de entre otras múltiples realidades, así como la existencia de varias realidades diferentes como ser el mundo de los sueños, el arte, la religión entre otros; aunque la más importante -desde su punto de vista-, es el mundo intersubjetivo de la vida cotidiana (el mundo de la vida), que representa el "arquetipo de nuestra experiencia de la realidad. Todos los demás ámbitos de significado pueden considerarse modificaciones de aquella" (Ritzer, 1993, 370).

El mundo de la vida es aquel de la cotidianeidad y en donde cualquier sujeto realiza una experiencia que es necesariamente única, por el hecho de que lleva sobre sí una biografía personal -situación biográfica-, una especie de itinerario experiencial en donde se mezclan aspectos adquiridos en la socialización primaria y la socialización secundaria; además de expectativas y deseos íntimos desde los cuales se piensa y actúa. Algo así como lo que Schutz, denomina los "reservorios de saber".

La fenomenología como corriente filosófica abre un camino para la "comprensión y análisis del conocimiento del mundo que tienen los sujetos y su punto de partida es que no se puede comprender al hombre y al mundo, sino es a partir de la facticidad, es decir, de los hechos" (Rizo, 2006, 1).

En términos estrictamente metodológicos, la fenomenología es una filosofía trascendental que pone en suspenso -para comprenderlas-, las afirmaciones de la actitud natural; pero es también una filosofía para la cual el mundo está "ya ahí", antes de cualquier reflexión.

Schutz propone que el mejor enfoque para el estudio de lo social, es posible sólo a través de una fenomenología de la actitud natural en la comprensión de que el mundo de la vida

\footnotetext{
${ }^{9}$ Precisamente, aquí estriba la diferenciación de las orientaciones fenomenológicas tanto de Edmund Husserl y Alfred Schutz; pues el primero identifica el ego trascendental (reino de la conciencia como procesos mentales de la subjetividad individual) como su preocupación central; el segundo, se ocupa más bien por construir una fenomenología para analizar el mundo intersubjetivo, el mundo social exterior; en el mundo de la vida, en las relaciones-nosotros y en las relaciones-ellos. No obstante a ello, Schutz no abandona del todo el interés tradicional de la fenomenología por la subjetividad aunque un tanto alejada de la subjetividad individual de Husserl-; pero orientada hacia la intersubjetividad.
}

cotidiana posee una estructura provista de sentido. Esto, en razón a que la "definición del mundo del individuo surge de su subjetividad, sedimentada y estructurada de manera exclusiva... aunque el individuo define su mundo desde su propia perspectiva, es, no obstante, un ser social, enraizado en una realidad intersubjetiva" (Schutz, 1974, 19).

Así, el planteamiento central de la sociología fenomenológica de Schutz es el concepto de Intersubjetividad, en tanto que el "mundo de la vida diaria en el cual nacemos es, desde el primer momento, un mundo intersubjetivo......La vida no considera el problema de cómo accedo a la mente del otro" (Schutz, 1974, 19).

El lebenswelt ${ }^{10}$ o mundo de la vida "presupone la intersubjetividad como una realidad obvia de nuestro mundo" (Schutz, 1974, 19).

De tales definiciones se deduce que ese mundo intersubjetivo no es un mundo particular o privado. Al contrario, es un mundo común a todos -mundo social-y "existe porque vivimos en él como hombres entre hombres, con quienes nos vinculan influencias $y$ labores comunes, comprendiendo a los demás y siendo comprendidos por ellos" (Ritzer, 1997, 268); por lo mismo, necesariamente existe en la cotidianeidad, en el presente vivido, en el diálogo, la comunicación y la interacción simultánea que realizan los unos y los otros.

Precisamente, esa simultaneidad constituye la esencia de la intersubjetividad, "significa que capto la subjetividad del alter ego al mismo tiempo que vivo en mi propio flujo de conciencia.... $Y$ esta captación en simultaneidad del otro, así como su captación recíproca de mí, hacen posible nuestro ser en conjunto con el mundo" (Ritzer, 1993, 376).

Respecto a la interacción, Schutz no estaba interesado en la interacción de tipo físico, sino más bien en aquel modo o aquellos modos, en el

\footnotetext{
10 El Lebenswelt, o mundo de la vida, es un término derivado originalmente del planteamiento de Husserl, pero al que Schutz la aplica para referirse al mundo en el que la intersubjetividad y la utilización de tipificaciones tienen lugar. Constituye ese mundo donde las personas actúan con la actitud natural, donde dan por establecido que el mundo existe y no dudan de esa realidad, hasta que surgen situaciones problemáticas. De la revisión bibliográfica, es posible hallar ciertos sinónimos empleados para dar cuenta del término Lebenswelt, tales como ser: "mundo-vida"; "mundo de la vida diaria"; "mundo vital"; "mundo vivido"; "realidad mundana"; "realidad eminente de la vida del sentido común".
} 
que las conciencias de los actores vienen a interactuar y a comprenderse recíprocamente y, sobre todo, en la manera en que se relacionan intersubjetivamente tales conciencias en el mundo social. Esto en razón a que la "conciencia del hombre está determinada por su ser social" (Berger; Luckmann, 2001, 18).

Asume con ello, que el conocimiento es intersubjetivo (social) debido principalmente a la existencia de perspectivas recíprocas, mediante las cuales, se da por supuesto que las otras personas existen y que los objetos son susceptibles de ser conocidos o aprehendidos; dejando claro que no necesariamente adquieren una significatividad uniforme para todos un mismo objeto analizado; al contrario, ese mismo objeto puede adquirir significados diferentes.

Tal disyuntiva -siguiendo a Schutz-, es plenamente superable en el mundo social, en tanto es posible recurrir por un lado, a la idealización de la intercambiabilidad de los puntos de vista que permite a las personas situarse en el lugar de los otros (empatía) para ver las cosas -el objeto- tal como la aprecian y, por otro, la idealización de la congruencia del sistema de relevancia que implica el abandono progresivo de las diferencias - puntos de vista particulares sobre los objetos-, aceptando que los objetos cuentan con ciertas precisiones $y$ definiciones generales para todos -uniformidad mínima o básica de criterios-, que proceden bajo el supuesto que tales definiciones son idénticas.

Finalmente, debemos mencionar que el conocimiento -concebido bajo estos preceptostiene origen social, toda vez que las personas van creando su propio conocimiento a partir de acervos comunes de conocimiento adquiridos a través de la interacción social con los otros; el cual se halla condicionado a una especie de distribución social del conocimiento que tiende a variar y diferenciarse de acuerdo a la posición que ocupan las personas al interior de la estructura social.

\subsection{Orientaciones Críticas para el Abordaje de la Realidad Social}

El abordaje de la realidad social, requiere de aquellos discursos de los actores sociales que revelan el sentido subjetivo atribuido a la acción social; pues gran parte de nuestra experiencia en el mundo de la vida (lebenswelt) -siguiendo a Schutz-, es comunicable (dejando claro que no necesariamente puede expresarse en términos de verbalización); por lo que resulta imposible renunciar a la importancia capital del lenguaje y las tipificaciones, como mediadores en los términos de comunicación de experiencias.

De hecho, las personas permanentemente utilizan tipificaciones para analizar el mundo social y la comprensión de los factores sociales de la vida cotidiana en la cual se hallan inmersos.

Las "tipificaciones se derivan de factores sociales y son socialmente aprobadas, a la vez que permiten a las personas actuar sobre la base de su cotidianeidad" (Ritzer, 1993, 390); pero cuando estos factores sociales se convierten en situaciones problemáticas, ocasionan el progresivo abandono de tales tipificaciones, dando lugar al desarrollo y creación mental de nuevas fórmulas y/o maneras (recetas) para manejar o tratar las situaciones del mundo social.

Dicho de otra forma, cuando el acervo de conocimiento actualmente disponible, ya no es suficiente para manejar las situaciones del mundo social, las personas deben crear e incorporar nuevas tipificaciones (recetas), que le permitan afrontar las circunstancias imprevistas no convencionales producto de la dinámica social.

Schutz consideraba que las tipificaciones adquieren una diversidad de formas, a tal punto que siempre que hacemos uso del lenguaje -que es el "medio tipificador por excellence"- (Ritzer, 1993, 374), las personas continuamente tipifican las cosas, los objetos, las acciones y reacciones, con la única intención de asignarle cierto sentido al mundo social.

Las tipificaciones existen en la sociedad y es precisamente ahí, donde las personas adquieren y almacenan tipificaciones a través del proceso de socialización y que constituye además, una especie de herramientas tradicionales $y$ habituales para la vida social; pues se derivan de la sociedad y se hallan socialmente aprobadas en la vida cotidiana, que "por sobre todo, es vida con el lenguaje -y las tipificaciones- que comparto con mis semejantes y por medio de él. Por lo tanto, la comprensión del lenguaje es esencial para cualquier comprensión de la realidad de la vida cotidiana" (Berger; Luckmann, 2001, 55). 
En esa dirección, no es equívoco señalar que el lenguaje representa la condición previa de la cultura; toda vez que, aquello que efectivamente nos distingue de las especies inferiores no es la inteligencia como tal, sino esa capacidad de hablar y de construir sistemas y esquemas simbólicos como signos representativos de significación y objetivación de objetos, cosas y fenómenos de la realidad, que "marca las coordenadas de mi vida en la sociedad y llena esa vida de objetos significativos" (Berger; Luckmann, 2001, 39).

Por lo mismo, el lenguaje es considerado como aquel "órgano de transformación simbólica de la realidad $y$, en definitiva, el modo de existencia del pensamiento humano" (Ortíz-Osés; Lanceros, 2006, 318); además de constituir el órgano que posibilita la instalación humana en la cultura.

Así, el lenguaje representa ese sistema de signos vocales más importante de la sociedad humana, que descansa en la capacidad intrínseca de la expresividad vocal del organismo humano y que más tarde, se convierte en expresiones lingüísticas en tanto se integran dentro de un sistema de signos accesibles objetivamente con capacidad de comunicar significados y significantes.

De esta manera, el lenguaje como expresión lingüística que comunica significados y significantes, es "capaz de transformarse en depósito objetivo de vastas acumulaciones de significado y experiencia, que puede preservar a través del tiempo y transmitir a las generaciones futuras" (Berger; Luckmann, 2001, 56).

Ahora bien, con base en las orientaciones teóricas planteadas, podemos señalar que la realidad que denominamos social, no es el resultado de una simple percepción de lo que nos es externo, sino que es resultante de una construcción socialmente admitida como tal; el cual se logra a través de evidencias empíricas de aquello que se considera como algo real a través de sus efectos y que es ampliamente compartida con los otros.

Precisamente, la "experiencia más importante que tengo de los otros se produce en la situación cara a cara, que es el prototipo de la interacción social" (Berger; Luckmann, 2001, 46); donde es posible compartir el presente vivido con los otros, produciéndose un intercambio continuo de expresividades intencionales dirigidas por los actores de manera simultánea.

Cabe también tener presente, que toda actividad interpretativa no constituye sino un paso más hacia un horizonte de objetivación de los fenómenos sociales, subentendiendo que una interpretación puede siempre ser corregida por otra.

Tampoco es inútil recordar que la interpretación que se da a conocer, tiene su base de sustentación interna en su propia coherencia y en su unidad lógica, en su capital de datos y en su adecuado tratamiento; en su rigor operativo, en su capacidad heurística demostrativa y en su contrastabilidad, la cual siempre tiende a reflejar esfuerzos intelectuales por constituir progresivamente un conocimiento que se prolonga hacia una verdad, que dada sus características y naturaleza, no puede ser conquistada en su totalidad.

De hecho, "juzgamos el conocimiento por aquello que puede mostrar (en lo teórico y empírico) y no por su adecuación respecto de algún patrón externo" (Follari, 2000, 104-105).

Cuando planteamos que el conocimiento no puede ser alcanzado en su totalidad, nos respaldamos en el hecho que ese conocimiento posee un carácter histórico y cultural que refleja el modo de ser del hombre, fundados en sus necesidades, intereses y fines (pensamientos, acciones e intenciones). Por lo que su revelación, no es sólo un producto cognoscitivo, sino que contempla todas aquellas actuaciones prácticas y/o transformadoras de los hombres.

El solo hecho de conocer los fenómenos sociales, exige asumir un carácter constitutivo crítico en la ciencia con capacidad de generar procesos de reflexividad, considerada como aquella "cualidad del discurso científico, que no sólo ha de hacer ser conscientes los presupuestos sociales que son el resultado de la investigación de un objeto, sino que también tiene que traer a la consciencia aquellos presupuestos sociales que son el origen de la labor de construcción de ese objeto, $y$ que se presentan en forma de opciones $y$ tensiones ideológicas y metodológicas de esa labor" (Bourdieu, 2001, 10-11), pues de lo que se trata, es de reivindicar el primado de las relaciones. 
Lo real bajo ninguna circunstancia se identifica con las sustancias, sino fundamentalmente con lo "que existe en el mundo social... son relaciones, no interacciones o vínculos subjetivos entre los agentes, sino relaciones objetivas que existen independientemente de las conciencias individuales" (Bourdieu, 2001, 14).

Por lo tanto, los elementos individualizados sólo tienen propiedades en virtud de las relaciones que mantienen con otros en un sistema o en un campo social; toda vez que "lo real es relacional, por eso mismo es necesario pensar relacionalmente lo real, como el primer precepto metodológico con el que combatir la inclinación a pensar el mundo social de manera realista o sustancialista" (Bourdieu, 2001, 14).

Dicho de otra forma, aquellas partes o elementos particulares, tienen sentido (propiedades) en tanto mantienen relaciones con las otras partes o elementos en un sistema de relaciones establecidos en el campo social, que es una especie de "construcción analítica mediante la que designa un conjunto específico y sistemático de relaciones sociales; es decir, se trata de una especie de sistema, definible sólo históricamente, que permite trasladar al análisis social la dinámica de relaciones que se desarrollan en la práctica" (Bourdieu. 2001:15).

Convencionalmente se ha dado por supuesto que la realidad social es fácilmente comprensible, generalizable a partir de sus datos y descrita tal cual se presenta, recurriendo para ello -las más d las veces-, a la estadística como estrategia conducente a mostrar deliberadamente -aunque no siempre consciente-, cierta objetividad sobre esa realidad, producto de esa representación o preconstrucción que tiene lugar en la cotidianeidad.

Una posibilidad de superar este procedimiento tradicional de aproximación a la realidad, pasa por "romper con las preconstrucciones del sentido común, esto es, con la realidad tal como ella misma se presente, de cara a construir sus propios objetos, incluso al riesgo de que parezca violentarse a esa realidad, de ajustar los datos a las necesidades de la construcción científica, o, más simplemente, de enfrentarse a una especie de vacío empírico, como cuando la información necesaria es incompleta o imposible de comparar, o, peor aún, cuando no existe y no puede ser producida" (Bourdieu, 2001, 70).
Cabe también mencionar que uno de los principales obstáculos al desarrollo y progreso de las Ciencias Sociales, queda reservada a la tradición academicista de obediencia y cumplimiento de aquellas reglas científicas definidas por la doxa metodológica, que a decir de muchos, otorgan rigurosidad científica a los hallazgos obtenidos, aceptando con cierta facilidad tales construcciones, además de la aprobación plena de la comunidad académica.

En contrapartida, cuando surgen otras formas de hacer investigación, cuya intencionalidad sea ir más allá de lo preceptuado por esa doxa metodológica rígida y positivista, que genera rupturas estructurales con aquellas falsas evidencias obtenidas de la realidad y el sentido común, tiende siempre a ser considerado como un peligro a la construcción del edificio del pensamiento científico.

De hecho, el objeto de la Ciencia Social es una realidad que "engloba todas las luchas, individuales $y$ colectivas, que apuntan a conservar o a transformar la realidad, y en particular aquellas cuyo asunto en juego es la imposición de la definición legítima de la realidad y cuya eficacia estrictamente simbólica puede contribuir a la conservación o a la subversión del orden establecido, es decir, de la realidad" (Bourdieu, 2007, 227).

Más aún, si aceptamos categóricamente que el conocimiento se "halla distribuido socialmente y el mecanismo de esta distribución puede constituirse en objeto de una disciplina sociológica" (Berger; Luckmann, 2001, 31).

Sin duda, estas formas de oposición o antinomias lejos de mostrarse como obstáculos al progreso científico, existen y en algún modo coadyuvan a su desarrollo; pues construyen la realidad social o más precisamente, construyen los instrumentos de construcción de la realidad (teorías, esquemas conceptuales, datos); definiendo lo visible y lo invisible, encubriendo y revelando aquello pensable y lo impensable; en razón a que las estructuras sociales "son el producto del desarrollo histórico y de las luchas históricas que deben ser analizadas si uno quiere evitar la naturalización -sin cuestionamientosde dichas estructuras" (Bourdieu, 2001, 75). 


\section{Reflexiones finales}

La construcción de conocimiento en las Ciencias Sociales, posee sin duda alguna, un carácter inacabado dada su provisoriedad; pues queda claro que existe siempre algo radicalmente incompleto en este tipo de conocimiento, cuyo estudio se halla precisamente orientado al análisis de los fenómenos sociales.

Arribamos a esta afirmación, en virtud de que los fenómenos sociales en general, tienden a asemejarse a periodicidades irrepetibles no sólo por sus actores que en ella interactúan, sino básicamente por la delimitación del tiempoespacio; sumados al hecho de que son inestables, complejas, dinámicas y contradictorias, que dificultan aún más, las posibilidades de su aprehensión e interpretación.

Debe agregarse a ello, que la concepción misma de la realidad del mundo tiene un carácter de comprensión insuficiente y hasta en algunos casos sesgada, en virtud a que se le atribuye sólo a los sentidos, la posibilidad de abordar la realidad (objetos), pero que deja de lado a aquellas otras formas, estados o situaciones existentes en la realidad y que no son accesibles a los sentidos orgánicos; al extremo tal, que ni aún las ciencias ni la reflexión filosófica -concretamente la gnoseología-, han logrado enfocar con cierta precisión e integralidad, la naturaleza y complejidad de sus componentes.

$\mathrm{Si}$ bien, por un lado, esto trae consigo profundas preocupaciones a la hora de realizar abordajes sobre la realidad y, concretamente sobre los fenómenos sociales; por otro, tiende a generar interesantes desafíos y puntos de partida orientadores en su análisis, ya que permiten comprender que éstos fenómenos difícilmente pueden ser similares; aún si contaran con características morfológicas comunes (comprensión de la totalidad del objeto), el análisis de aquellos aspectos que a simple vista no pueden ser apreciados (circunstancias, intencionalidades, orientaciones propias de los actores) y, quizá la más importante, los fenómenos sociales nunca se repiten tal como ya han ocurrido, pues los actores, las circunstancias, intenciones $\mathrm{y}$ orientaciones que guiaron la acción ya no son los mismos (carácter histórico).

Dicho de otro modo, tanto las acciones como los hechos, difícilmente pueden ser similares, aún si contaran con características morfológicas de estados comunes.

Otro aspecto a considerarse estriba en el hecho de que la naturaleza de los fenómenos sociales y la realidad de la vida en general que nos circunda y en las que el cientista social se halla inmerso en su especificidad, obligan a desplegar esfuerzos cognitivos de comprensión, que vayan más allá de los aspectos visibles de la acción (objetividad) hasta tocar aquello que la inspira, vale decir su sentido subjetivo. Dicha tarea pasa necesariamente por retomar en el marco de una opción teórica determinada, múltiples elementos que consideren directamente aquellos planos de la empiria de la vida social.

Ello sin duda, resulta ser altamente gratificante para los cientistas sociales, pues se hallan ante la imperiosa necesidad de dar cuenta de una realidad compartida que es particular y específica, pero al mismo tiempo cambiante y dinámica.

Por otro lado, abre la posibilidad de que esta realidad pueda ser comprendida, interpretada y captada desde la propia experiencia, aunque no del todo individual; esto en razón a que la experiencia misma del mundo, es una genuina experiencia existencial colectiva de ese mundo cotidiano; que se construye y reconstruye, se configura y reconfigura en la dinámica de esas interacciones situadas $\mathrm{y}$ fechadas, en las significaciones y resignificaciones colectivas. Es ahí precisamente, donde radica su valía y trascendencia.

Por último, la intención de comprender los fenómenos sociales, exige ir más allá de lo objetivado (lo real, lo concreto observado a primera vista), ya que en muchos casos, se requiere apelar a la intersubjetividad -que implica la posibilidad de reconocer que en esa realidad estudiada nos encontramos inmersos, vivimos en ella, pero también nosotros construimos esa realidad compartida, la cual nos es común a todos porque compartimos el mismo tiempo y espacio con otros-, para entender las razones o circunstancias que explican las acciones que en ella se observan. 


\section{Referencias}

Adorno, T. W. (2001). Epistemología y Ciencias Sociales. Madrid: Ed. Frónesis Cátedra Universitat de Valencia.

Alexander, J. C. (1992). Las Teorías Sociológicas Desde la Segunda Guerra Mundial. Barcelona: Gedisa. Berger, P. L.; Luckmann, T. (2001). La Construcción Social de la Realidad. Buenos Aires: Amorrortu.

Bourdieu, J. P. (1997). Razones Prácticas Sobre la Teoría de la Acción. Barcelona: Anagrama.

- (2003). El Oficio de Científico. Ciencia de la Ciencia y Reflexividad Curso del College de France 2000-2001.

Barcelona: Anagrama.

- (2007). El Sentido Práctico. Buenos Aires: Siglo XXI.

- (2001). Poder, Derecho y Clases Sociales. Bilbao: Desclée de Brouwer.

Covarrubias, V. F. 0. Las Herramientas de la Razón. La Teorización Potenciadora Intencional de Procesos Sociales. Ed. SEP -UPN. México. Citado por C. E. Massé Narvaez en Del positivismo Disciplinario y el Racionalismo Crítico a la Epistemología Crítica Dialéctica con Base en la Totalidad, Como Método Alternativo de la Investigación Científica. Cinta de Moebio (Revista Electrónica de Epistemología de Ciencias Sociales). Facultad de Ciencias Sociales. (Septiembre №11). Universidad de Chile. Santiago - Chile. 2001. (Soporte Electrónico). Se puede encontrar en: www.moebio.uchile.clhttp://www.facso.uchile.cl/publicaciones/moebio/guide.html

Follari, R. (2000). Epistemología y Sociedad. Santa Fe: Homo Sapiens.

Massé Narvaez, C. E. (2001). Del Positivismo Disciplinario y el Racionalismo Crítico a la Epistemología Crítica Dialéctica con Base en la Totalidad, como Método Alternativo de la Investigación Científica. Cinta de Moebio (Revista Electrónica de Epistemología de Ciencias Sociales). Universidad de Chile. Santiago - Chile. Se puede encontrar en: www.moebio.uchile.cl http://www.facso.uchile.cl/publicaciones/moebio/guide.html

Ortiz-Osés, A.; Lanceros, P. (2006). Diccionario Interdisciplinar de Hermenéutica. Bilbao: Universidad de Deusto.

Ritzer, G. (1993). Teoría Sociológica Clásica. Madrid: McGraw-Hill.

- (1997). Teoría Sociológica Contemporánea. México D.F.: McGrawhill/Interamericana.

Rizo G., M. (2006). Intersubjetividad, Vida Cotidiana y Comunicación. Revista Científica de Comunicología, Indicios y Conjeturas, 5.

Schutz, A. (1974). El Problema de la Realidad Social. Buenos Aires: Amorrortu.

Szilasi, W. (1973). Introducción a la Fenomenología de Husserl. Buenos Aires: Amorrortu. 